\title{
Spontaneous synchronous bilateral hemothorax as the only finding in primary pleural angiosarcoma: a case report and a literature review
}

\author{
Guido Levi $^{1}$, Nicla Orzes ${ }^{1}$, Silvia Uccelli ${ }^{1}$, Francesca Cettolo ${ }^{1}$, Marianna Arici ${ }^{1}$, Manuela Ciarfaglia ${ }^{1}$, \\ Simona Fisogni ${ }^{2,3}$, Giampietro Marchetti ${ }^{4}$, Chiara Rocchetti ${ }^{1}$ \\ ${ }^{1}$ Respiratory Medicine Unit, Department of Clinical and Experimental Sciences, University of Brescia; ${ }^{2}$ Department of \\ Molecular and Translational Medicine. University of Brescia; ${ }^{3}$ Pathology Unit, ASST Spedali Civili di Brescia; \\ ${ }^{4}$ Department of Pneumology, ASST Spedali Civili di Brescia, Italy
}

\begin{abstract}
Angiosarcoma is a rare malignancy of vascular origin, mostly originating from skin, soft tissues, and breast, but rarely also from the pleura. We present the case of a 55-year-old man who referred to our hospital for a spontaneous bilateral hemothorax. The CT
\end{abstract}

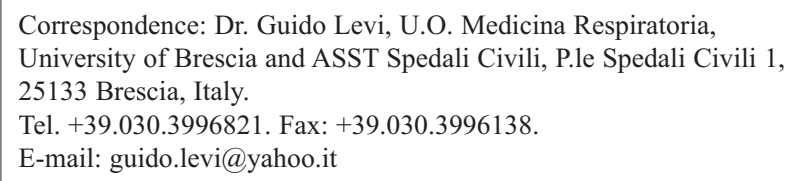

Authors' contributions: All authors contributed to this article by collecting data, drafting the article, approving its final version and agreeing to be accountable for all aspects of the work.

Conflict of interests: None to declare.

Availability of data and materials: All data underlying the findings are fully available.

Ethics approval and consent to participate: No ethical committee approval was required for this case report by the Department, because this article does not contain any studies with human participants or animals. Informed consent was obtained from the patient included in this study.

Funding: None received.

Acknowledgments: The authors wish to thank all Pleural-Hub affiliates for their continuous support, interest and enthusiasm.

Received for publication: 20 July 2020.

Accepted for publication: 25 November 2020.

${ }^{\circ}$ Copyright: the Author(s), 2021

Licensee PAGEPress, Italy

Monaldi Archives for Chest Disease 2021; 91:1520

doi: 10.4081 monaldi.2021.1520

This article is distributed under the terms of the Creative Commons Attribution Noncommercial License (by-nc 4.0) which permits any noncommercial use, distribution, and reproduction in any medium, provided the original author(s) and source are credited. angiography did not show any source of active bleeding; plus, no pleural or lung masses were observable. Cytological and microbiological analyses made on a sample of pleural fluid resulted negative. Despite numerous blood transfusions and thoracenteses, the patient deceased from hemorrhagic shock ten days later and the diagnosis of primary pleural epithelioid angiosarcoma was obtained only by autopsy. Additionally, we present a review of the literature about primary pleural angiosarcomas.

\section{Introduction}

Angiosarcoma (AS) is a rare malignancy of vascular origin, representing about $1 \%$ of all the soft tissues' sarcomas. It most often occurs in the skin, soft tissues, and breast. Its presentation and behavior differ depending on the location involved. ASs can be divided into four groups: cutaneous AS associated or unassociated with lymphedema, AS of the breast, and AS of deep soft tissue [1].

Pleurae can be affected by metastases or, rarely, as the first site of malignancy. Histologically, two variants are observable: the morphology of the pleural angiosarcoma can be spindle-shaped or can be of epithelioid type. In particular, the epithelioid type accounts for $75 \%$ of the pleural ASs and it is related to a worse prognosis [2]. We present the case of a 55-year-old Caucasian male affected by an extremely aggressive form of pleural epithelioid AS, which presented itself as spontaneous bilateral hemothorax without macroscopic or radiological pleural lesions.

\section{Case Report}

A 55-year-old Caucasian male was admitted to a local hospital because of dyspnea; he had no significant respiratory disease, but 16 months earlier he underwent a percutaneous transluminal coronary angioplasty with stent placement due to coronary artery disease; furthermore, no trauma was reported. Blood tests showed anemia (hemoglobin $8.6 \mathrm{~g} / \mathrm{dL}$ ) and leukocytosis. Electrocardiogram and echocardiogram showed no significant findings. At the chest X-ray bilateral pleural effusion was observed, confirmed by the subsequent CT-scan (Figure 1); the liquid had a density of 20-25 Hounsfield Units, consistent with blood. No pleural lesions, lymphadenopathies or metastases were observable. The patient was then transferred to our hospital in order to perform a CT angiography, which showed no active bleeding. Dual antiplatelet therapy (clopidogrel and acetyl-salicylic acid) was promptly 
discontinued and antibiotic therapy with Azithromycin and Cefotaxime was started. A chest tube was placed in the left pleural space: 4 liters of hematic fluid (hemoglobin $7.0 \mathrm{~g} / \mathrm{dL}$ ) were drained, with partial regression of dyspnea. Cytological and microbiological analyses resulted negative. Multiple blood transfusions were performed, and tranexamic acid was administered. Six days later, clinical conditions severely deteriorated: a new CT showed increasing bilateral pleural effusion, yet no active bleeding and no lesions were observed (Figure 1). Again, no malignant cells were found at the cytological analysis. Given the rare nature of the disease, the case was thoroughly discussed both internally and on "Pleural Hub" [3], a Facebook group which connects many Italian physicians in order to discuss complex clinical cases, mainly about pleural pathology. Many hypotheses were done, especially about a malignant etiology of the hemothorax, such as hemangioendothelioma, primary or secondary AS or a hematologic malignancy. A surgical revision or a medical thoracoscopy were suggested, but the precarious conditions of the patient did not allow any invasive procedures.

Despite maximum efforts, the patient deceased two weeks after the admission and afterwards an autopsy was performed. No macroscopic masses were observed. The final diagnosis, obtained via histological examination of both pleurae, was a bilateral epithelioid pleural AS, with lung, thoracic nodal and pericardial metastases (Figure 2).

\section{Discussion}

Spontaneous hemothorax is a subcategory of hemothorax that involves the accumulation of blood within the pleural space in the absence of trauma or other causes. The clinical presentation is variable, including a rapid progression of symptoms (e.g., dyspnea and chest pain) that can be life-threatening [4]

The primary treatment for AS, especially for localized lesions, is surgical resection. However, as it grows rapidly, very few cases can undergo surgery. Radiotherapy could play a beneficial role in the absence of diffuse lesions, while standard chemotherapy has few effects, and it is generally performed as a palliative treatment [2].

In our review of the literature, we found 34 cases of primary pleural ASs (Table 1). The average age at presentation is $65 \pm 12$ years and males are affected more frequently $(82 \%)$. To date, this is the only case in literature of a primary synchronous bilateral pleural AS with no macroscopic anatomical or radiological masses. In fact, in all the cases reviewed, pleural thickening or macroscopic pleural lesions or metastases are reported, as a suggestion to the diagnosis.

\section{Conclusions}

Since its clinical presentation is devious and its prognosis is often extremely poor, early recognition of this malignancy should be the most desirable target. It should always be suspected in the presence of spontaneous hemothorax, even if no lesions are observable at CT-scans. Unfortunately, performing biopsies is not always a feasible option. Few cases are reported in literature, and further studies and case reports are needed. Sharing these complex cases via simple online tools, such as Facebook groups like "Pleural Hub", may expedite diagnosis, even though palliative treatment may be the only available option.
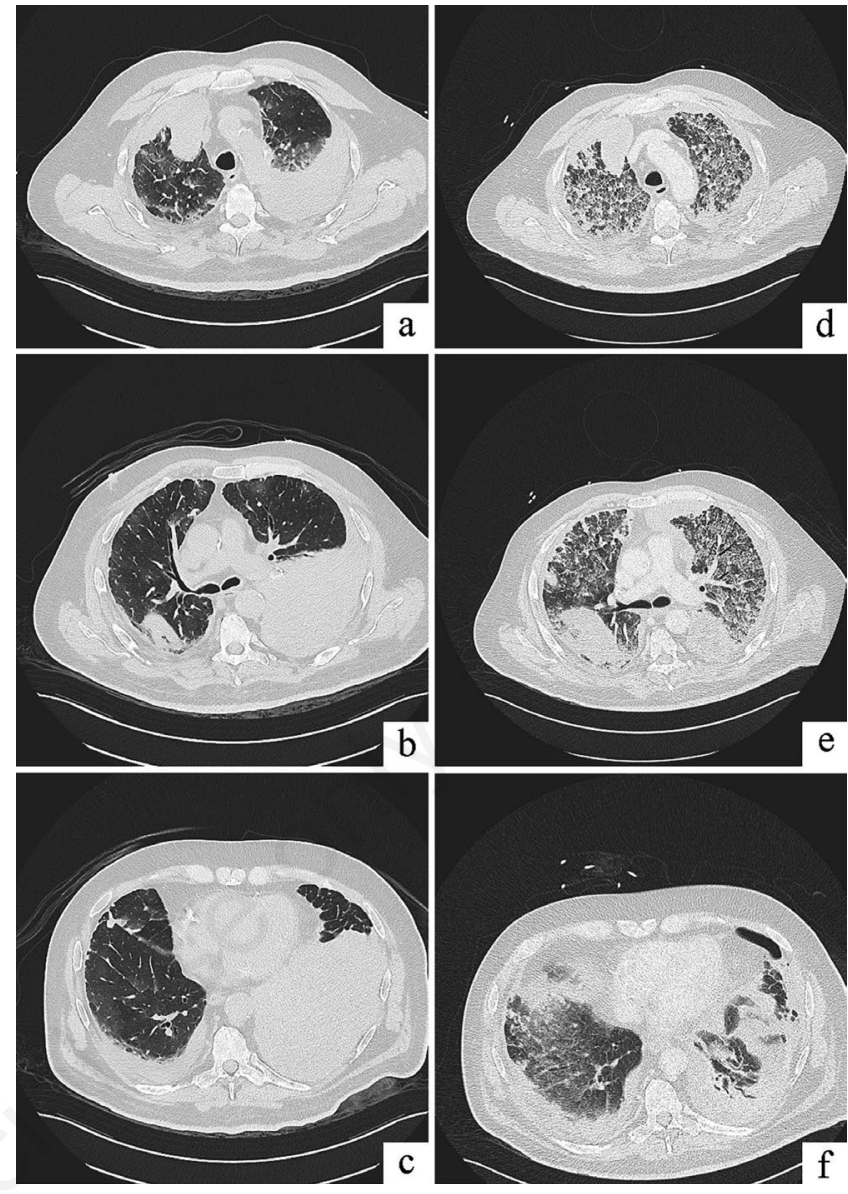

Figure 1. a,b,c) CT scan at the admission shows bilateral hematic pleural effusion and ground-glass alterations. d,e,f) CT scan performed after 6 days reveals increasing ground-glass alterations and bilateral pleural effusion, despite the chest tube.
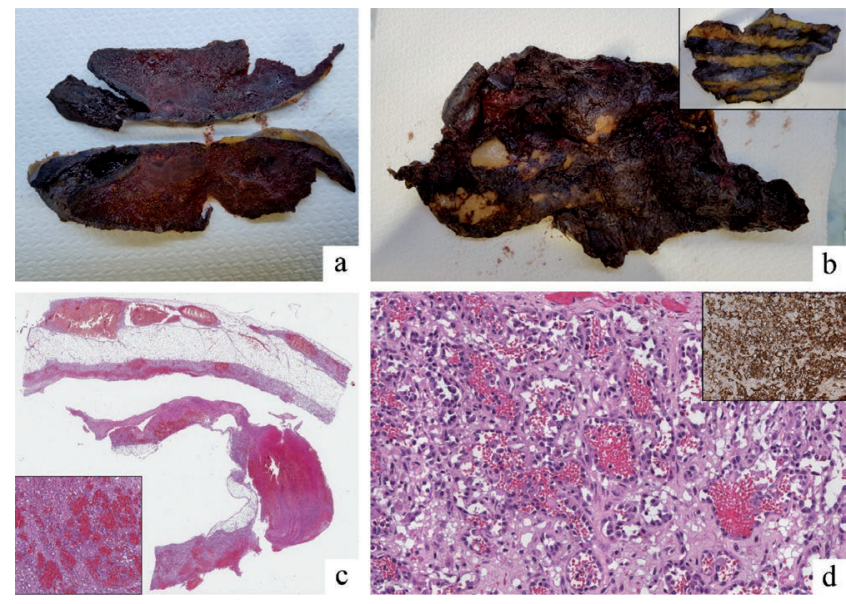

Figure 2. a) Severe hemorrhagic infarction of lung parenchyma with consolidated areas. The space between visceral and parietal pleura is filled with blood and fibrin. No space-forming masses are recognizable. b) Internal surface of parietal pleura with blood and fibrin stratification. External surface of parietal pleura (inset). c) Histological scan section of pathological parietal pleura; next to hemorrhagic areas, diffuse neoplastic proliferation with vascular forming architecture (inset). d) The neoplastic cells show epithelioid morphology. Diffuse positivity for vascular marker CD31 (inset). 
Table 1. Angiosarcoma cases in the literature.

\begin{tabular}{|c|c|c|c|c|c|c|}
\hline Author and Journal & Sex, age & $\begin{array}{l}\text { Clinical } \\
\text { presentation }\end{array}$ & $\begin{array}{l}\text { Lesions at chest } \\
\text { CT-scan }\end{array}$ & Thoracoscopy & $\begin{array}{l}\text { Extra-thoracic } \\
\text { metastases } \\
\text { at diagnosis }\end{array}$ & Treatment \\
\hline $\begin{array}{l}\text { Azzakhmam } \\
\text { Pan Afr Med J } \\
2019[2]\end{array}$ & $\mathrm{M}, 70$ & Dyspnea, ascites & $\begin{array}{l}\text { Right-sided pleural } \\
\text { thickening, } \\
\text { lymphadenopathy }\end{array}$ & $\begin{array}{l}\text { Biopsies from the } \\
\text { parietal pleura }\end{array}$ & No & No \\
\hline $\begin{array}{l}\text { Kubo } \\
\text { Respirol Case Rep } \\
2019 \text { [5] }\end{array}$ & $\mathrm{M}, 82$ & $\begin{array}{l}\text { Epigastric and } \\
\text { right-sided chest } \\
\text { pain }\end{array}$ & $\begin{array}{l}\text { Pleural thickening, } \\
\text { mass on the seventh } \\
\text { rib }\end{array}$ & $\begin{array}{l}\text { Not performed. } \\
\text { CT- guided } \\
\text { needle biopsy }\end{array}$ & Bones, liver & RT \\
\hline $\begin{array}{l}\text { Filippiadis } \\
\text { J Vasc Interv Radiol } \\
2018 \text { [6] }\end{array}$ & $\mathrm{M}, 74$ & $\begin{array}{l}\text { Right-sided } \\
\text { hemorrhagic pleural } \\
\text { effusion }\end{array}$ & $\begin{array}{l}\text { Nodular paraspinal } \\
\text { posterior chest } \\
\text { wall mass }\end{array}$ & Not diagnostic & - & Surgery \\
\hline $\begin{array}{l}\text { Durani } \\
\text { Respir Med Case Rep } \\
2018 \text { [7] }\end{array}$ & $\mathrm{M}, 62$ & $\begin{array}{l}\text { Right-sided pleural } \\
\text { effusion }\end{array}$ & $\begin{array}{l}\text { Ground-glass } \\
\text { pulmonary } \\
\text { nodules }\end{array}$ & Areas of nodularity & Bones & No \\
\hline $\begin{array}{l}\text { Cabibi } \\
\text { Diagn Pathol } \\
20178[8]\end{array}$ & $\mathrm{M}, 50$ & $\begin{array}{l}\text { Dyspnea, left-sided } \\
\text { pleural effusion }\end{array}$ & $\begin{array}{l}\text { Circumferential } \\
\text { pleural thickening } \\
\text { with pleural masses }\end{array}$ & $\begin{array}{l}\text { Not performed. } \\
\text { VATS biopsy }\end{array}$ & No & No \\
\hline $\begin{array}{l}\text { Miller } \\
\text { Am J Respir Crit Care Med } \\
2017 \text { [9] }\end{array}$ & $\mathrm{M}, 75$ & $\begin{array}{l}\text { Dyspnea and } \\
\text { left-sided pleural } \\
\text { effusion }\end{array}$ & $\begin{array}{l}\text { FDG-avid areas at } \\
\text { the PET-CT }\end{array}$ & Pleural mass & No & CTX \\
\hline $\begin{array}{l}\text { Yamaguchi } \\
\text { J Dermatol } \\
2017[10]\end{array}$ & $\mathrm{F}, 72$ & $\begin{array}{l}\text { Left-sided pleural } \\
\text { effusion }\end{array}$ & Pleural mass & Biopsy & Skin & CTX, RT \\
\hline $\begin{array}{l}\text { Patel } \\
\text { Chest } \\
2016[11]\end{array}$ & $\mathrm{M}, 72$ & $\begin{array}{l}\text { Spontaneous } \\
\text { pneumothorax }\end{array}$ & Not mentioned & $\begin{array}{l}\text { Not performed. } \\
\text { VATS biopsy }\end{array}$ & No & CTX \\
\hline $\begin{array}{l}\text { Panjwani } \\
\text { Egypt J Inter Med } \\
2016 \text { [12] }\end{array}$ & $\mathrm{M}, 76$ & $\begin{array}{l}\text { Right sided chest } \\
\text { pain, cough, } \\
\text { dyspnea }\end{array}$ & $\begin{array}{l}\text { Nodular pleural } \\
\text { thickening and } \\
\text { pleural effusion }\end{array}$ & $\begin{array}{l}\text { Not performed. } \\
\text { VATS biopsy }\end{array}$ & No & Surgery \\
\hline $\begin{array}{l}\text { Matsuda } \\
\text { Pathol Int } \\
2015 \text { [13] }\end{array}$ & $\mathrm{M}, 87$ & Anemia & $\begin{array}{l}\text { Nodular mass } \\
\text { on the right pleura }\end{array}$ & Not performed & - & - \\
\hline $\begin{array}{l}\text { Zhang } \\
\text { Int J Clin Exp Pathol } \\
2015 \text { [14] }\end{array}$ & $\mathrm{M}, 76$ & Cough & $\begin{array}{l}\text { Left pleural mass, } \\
\text { pleural thickening }\end{array}$ & Not performed & No & Surgery \\
\hline $\begin{array}{l}\text { Onur } \\
\text { J Pak Med Assoc } \\
2013 \text { [15] }\end{array}$ & F, 79 & Dyspnea & $\begin{array}{l}\text { Cystic and cavitary } \\
\text { lesions, left pleural } \\
\text { thickening }\end{array}$ & Not performed & No & No \\
\hline $\begin{array}{l}\text { Quesada } \\
\text { Ann Clin Lab Sci } \\
2013 \text { [16] }\end{array}$ & F, 58 & Dyspnea, fever & Pleural nodules & $\begin{array}{l}\text { Not performed. } \\
\text { VATS biopsy }\end{array}$ & - & CTX \\
\hline $\begin{array}{l}\text { Abu-Zaid } \\
\text { Case Rep Pulmonol } \\
2013 \text { [17] }\end{array}$ & $\mathrm{M}, 63$ & $\begin{array}{l}\text { Chest pain, dyspnea, } \\
\text { and hemoptysis }\end{array}$ & $\begin{array}{l}\text { Right lung thickening } \\
\text { and calcifications, } \\
\text { diaphragmatic } \\
\text { pleural masses }\end{array}$ & $\begin{array}{l}\text { Not performed. } \\
\text { CT-guided } \\
\text { needle-core biopsy }\end{array}$ & No & Surgery \\
\hline $\begin{array}{l}\text { Chen } \\
\text { Interact Cardiovasc } \\
\text { Thorac Surg } \\
2013[18]\end{array}$ & $\mathrm{M}, 69$ & $\begin{array}{l}\text { Chest pain and } \\
\text { body weight loss }\end{array}$ & Pleural hematomas & $\begin{array}{l}\text { Lesions on the lower } \\
\text { pleural cavity }\end{array}$ & No & No \\
\hline $\begin{array}{l}\text { Tsubouchi } \\
\text { J Thorac Oncol } \\
2012[19]\end{array}$ & M, 72 & Dyspnea & Left pleural mass & $\begin{array}{l}\text { Not performed. } \\
\text { Ultrasound-guided } \\
\text { biopsy }\end{array}$ & Skin, lung & CTX \\
\hline $\begin{array}{l}\text { Lorentziadis } \\
\text { Ann Thorac Surg } \\
2012[20]\end{array}$ & $\mathrm{M}, 77$ & Dyspnea & Pleural thickening & Not diagnostic & - & Surgery \\
\hline $\begin{array}{l}\text { Kao } \\
\text { Diagn Pathol } \\
2011 \text { [21] }\end{array}$ & M, 49 & Chest pain, dyspnea & Pleural thickening & $\begin{array}{l}\text { Blood clots and right } \\
\text { pleural thickening }\end{array}$ & - & Surgery, CTX, RT \\
\hline
\end{tabular}


Table 1. Continued from previous page.

\begin{tabular}{|c|c|c|c|c|c|c|}
\hline Author and Journal & Sex, age & $\begin{array}{l}\text { Clinical } \\
\text { presentation }\end{array}$ & $\begin{array}{l}\text { Lesions at chest } \\
\text { CT-scan }\end{array}$ & Thoracoscopy & $\begin{array}{l}\text { Extra-thoracic } \\
\text { metastases } \\
\text { at diagnosis }\end{array}$ & Treatment \\
\hline $\begin{array}{l}\text { Otsubo } \\
\text { Nihon Hinyokika } \\
\text { Gakkai Zasshi } \\
2011[22]\end{array}$ & M, 79 & Hematuria, anemia & No & Not performed & $\begin{array}{l}\text { Adrenal glands, } \\
\text { bones, stomach, } \\
\text { urinary bladder }\end{array}$ & No \\
\hline $\begin{array}{l}\text { Miyazaki } \\
\text { Hum Pathol } \\
2011 \text { [23] }\end{array}$ & $\mathrm{M}, 68$ & Hoarseness & $\begin{array}{l}\text { Capsulated left } \\
\text { pleural mass }\end{array}$ & $\begin{array}{l}\text { Not performed. } \\
\text { CT-guided needle } \\
\text { biopsy not diagnostic }\end{array}$ & Right lung & - \\
\hline $\begin{array}{l}\text { Baisi } \\
\text { Interact Cardiovasc } \\
\text { Thorac Surg } \\
2011[24]\end{array}$ & M, 75 & Left-sided chest pain & $\begin{array}{l}\text { Pleural thickening, } \\
\text { pleural masses }\end{array}$ & $\begin{array}{l}\text { Multiple blood-filled } \\
\text { cysts }\end{array}$ & - & Surgery, CTX \\
\hline $\begin{array}{l}\text { Dainese } \\
\text { Pathol Res Pract } \\
2010 \text { [25] }\end{array}$ & $\mathrm{M}, 62$ & $\begin{array}{l}\text { Dyspnea, bilateral } \\
\text { hemothorax }\end{array}$ & No & $\begin{array}{l}\text { Pleural fibrin coating, } \\
\text { lung consolidation }\end{array}$ & No & No \\
\hline $\begin{array}{l}\text { Saitou } \\
\text { Kekkaku } \\
2009[26]\end{array}$ & M, 76 & $\begin{array}{l}\text { Cough, chest pain } \\
\text { and dyspnea }\end{array}$ & $\begin{array}{l}\text { Soft tissue mass, } \\
\text { pleural calcifications }\end{array}$ & $\begin{array}{l}\text { Not performed. } \\
\text { CT- and US-guided } \\
\text { biopsies not } \\
\text { diagnostic }\end{array}$ & Bones & No \\
\hline $\begin{array}{l}\text { Kurtz } \\
\text { Sarcoma } \\
2004[27]\end{array}$ & $\mathrm{M}, 61$ & $\begin{array}{l}\text { Massive bilateral } \\
\text { hemothorax }\end{array}$ & No & $\begin{array}{l}\text { Multiple pleural } \\
\text { septa }\end{array}$ & Skin, oral mucosa & - \\
\hline $\begin{array}{l}\text { Chen } \\
\text { Arch Pathol Lab Med } \\
2004 \text { [28] }\end{array}$ & M, 39 & $\begin{array}{l}\text { Right-sided chest } \\
\text { pain, dyspnea, cough }\end{array}$ & $\begin{array}{l}\text { Pleural thickening, } \\
\text { right-sided mass }\end{array}$ & Not performed & No & Surgery, CTX \\
\hline $\begin{array}{l}\text { Pramesh } \\
\text { Ann Thorac Cardiovasc } \\
\text { Surg } \\
2004 \text { [29] }\end{array}$ & M, 55 & $\begin{array}{l}\text { Chest pain, cough, } \\
\text { hemoptysis }\end{array}$ & $\begin{array}{l}\text { Right-sided mass } \\
\text { with calcifications }\end{array}$ & Not performed & - & Surgery \\
\hline $\begin{array}{l}\text { Kimura } \\
\text { Pathol Int } \\
2003[30]\end{array}$ & M, 70 & $\begin{array}{l}\text { Intermittent } \\
\text { episodes of } \\
\text { memory loss, } \\
\text { headache }\end{array}$ & $\begin{array}{l}\text { Mass in the left } \\
\text { parietal lobe of the } \\
\text { brain, right-sided } \\
\text { thoracic mass }\end{array}$ & Not performed & Brain & No \\
\hline $\begin{array}{l}\text { Roh } \\
\text { J Korean Med Sci } \\
2001[31]\end{array}$ & F, 34 & Dyspnea, chest pain & Pleural thickening & Not performed & No & Surgery, CTX \\
\hline $\begin{array}{l}\text { Zhang } \\
\text { Hum Pathol } \\
2000[32]\end{array}$ & $\begin{array}{l}\mathrm{M}, 53 \\
\mathrm{~F}, 62 \\
\mathrm{M}, 66 \\
\mathrm{M}, 45 \\
\mathrm{M}, 60\end{array}$ & $\begin{array}{l}\text { Pleural effusion } \\
\text { Pleural effusion, ascit } \\
\text { Pleural effusion } \\
\text { Recurrent pleural eff } \\
\text { Bloody pleural effusic }\end{array}$ & $\begin{array}{l}\text { Pleural thickening } \\
\text { es } \\
\text { Pleural thickening } \\
\text { usion } \\
\text { in }\end{array}$ & $\begin{array}{l}\text { Not mentioned } \\
\text { Pleural thickening } \\
\text { Not mentioned } \\
\text { Pleural thickening } \\
\text { Pleural thickening }\end{array}$ & $\begin{array}{l}\text { Not mentioned } \\
- \\
\text { Not mentioned } \\
\text { Not mentioned }\end{array}$ & $\begin{array}{ll}- & \\
- & \text { Surgery } \\
- & \\
- & - \\
- & -\end{array}$ \\
\hline $\begin{array}{l}\text { Alexiou } \\
\text { Eur J Cardiothorac Surg } \\
1998 \text { [33] }\end{array}$ & F, 57 & $\begin{array}{l}\text { Massive bilateral } \\
\text { hemothorax }\end{array}$ & $\begin{array}{l}\text { Pleural bilateral } \\
\text { masses }\end{array}$ & Not performed & No & Surgery, RT \\
\hline
\end{tabular}

\section{References}

1. Moriya Y, Sugawara T, Arai M, et al. Bilateral massive bloody pleurisy complicated by angiosarcoma. Intern Med 2007;46: $125-8$.

2. Azzakhmam M, Elktaibi A, Reda El Ochi M, et al. Primary epitheloid angiosarcoma of the pleura: an exceptional tumor location. Pan Afr Med J 2019;33:327.

3. Pleural Hub Facebook Group. Accessed on: July 20, 2020. Available from: https://www.facebook.com/groups/pleural.hub/
4. Patrini D, Panagiotopoulos N, Pararajasingham J, et al. Etiology and management of spontaneous haemothorax. J Thorac Dis 2015 Mar; 7:520-6.

5. Kubo S, Kobayashi N, Kaneko A et al. Computed tomography imaging-based observation of the aggressive growth of angiosarcoma: a case study. Respirol Case Rep 2019;7:e00479.

6. Filippiadis DK, Kapetanakis EI, Spiliopoulos S, et al. Bleeding remission with microwave ablation in a transfusion-dependent patient with hemorrhaging angiosarcoma of the pleura. J Vasc Interv Radiol 2018;29:1298-300.

7. Durani U, Gallo de Moraes A, Beachey J, et al. Epithelioid 
angiosarcoma: A rare cause of pericarditis and pleural effusion. Respir Med Case Rep 2018;24:77-80.

8. Cabibi D, Pipitone G, Porcasi R, et al. Pleural epithelioid angiosarcoma with lymphatic differentiation arisen after radiometabolic therapy for thyroid carcinoma: immunohistochemical findings and review of the literature. Diagn Pathol 2017;12:60.

9. Miller R, Mudambi L, Vial MR, et al. Radiation-induced angiosarcoma as a cause of pleural effusion. Am J Respir Crit Care Med 2017;196:e10-1.

10. Yamaguchi K, Yoshino K, Imafuku K, et al. Case of primary pleural angiosarcoma with malignant seeding along the pleural tap tract. J Dermatol 2017;44:e75-6.

11. Patel MB, Munzer K, Dougherty M, et al. Pleural myiasis associated with pleural angiosarcoma. Chest 2016;149:e157-60.

12. Panjwani A, Singh I, Parvataneni N, Talukdar P. Spontaneous hemothorax: primary pleural epithelioid angiosarcoma. Egypt J Intern Med 2016;28:170-3.

13. Matsuda K, Yamaryo T, Akazawa Y, et al. Primary pleural angiosarcoma associated with pneumoconiosis: An autopsy case. Pathol Int 2015;65:603-7.

14. Zhang S, Zheng Y, Liu W, Yu X. Primary epithelioid angiosarcoma of the pleura: a case report and review of literature. Int J Clin Exp Pathol 2015;8:2153-8.

15. Onur ST, Günlüoglu Z, Dalar L, et al. Pleural angiosarcoma: a rare cause of spontaneous haemothorax. J Pak Med Assoc 2013;63:265-7.

16. Quesada A, Quesada J, Khalil K, et al. Morphoproteomic study of primary pleural angiosarcoma of lymphangioendothelial lineage: a case report. Ann Clin Lab Sci 2013;43:317-22.

17. Abu-Zaid A, Mohammed S. Primary pleural angiosarcoma in a 63-year-old gentleman. Case Rep Pulmonol.2013;2013: 974567.

18. Chen CY, Wu YC, Chou TY, Yang KY. Pleural angiosarcoma mimicking pleural haematoma. Interact Cardiovasc Thorac Surg 2013;17:886-8. .

19. Tsubouchi K, Yoshioka H, Ishida T. Significant response to gemcitabine monotherapy in primary pleural epithelioid angiosarcoma. J Thorac Oncol 2012;7:942-3.
20. Lorentziadis M, Sourlas A. Primary de novo angiosarcoma of the pleura. Ann Thorac Surg 2012;93:996-8.

21. Kao YC, Chow JM, Wang KM, et al. Primary pleural angiosarcoma as a mimicker of mesothelioma: a case report **VS**. Diagn Pathol 2011 30;6:130.

22. Otsubo S, Tanaka S, Kamiryo Y, et al. A case of primary pleural angiosarcoma metastasing to the bladder. Nihon Hinyokika Gakkai Zasshi 2011;102:686-90.

23. Miyazaki H, Goto A, Hino R, et al. Pleural cavity angiosarcoma arising in chronic expanding hematoma after pneumonectomy. Hum Pathol 2011;42:1576-9.

24. Baisi A, Raveglia F, De Simone M, Cioffi U. Primary multifocal angiosarcoma of the pleura. Interact Cardiovasc Thorac Surg 2011;12:1069-70.

25. Dainese E, Pozzi B, Milani M, et al. Primary pleural epithelioid angiosarcoma. A case report and review of the literature. Pathol Res Pract 2010;206:415-9.

26. Saitou M, Niitsuma K. A case of pyothorax-associated pleural angiosarcoma diagnosed by autopsy. Kekkaku 2009;84:531-4.

27. Kurtz JE, Serra S, Duclos B, et al. Diffuse primary angiosarcoma of the pleura: a case report and review of the literature. Sarcoma 2004;8:103-6.

28. Chen L, Shih HJ, Seguerra E Jr, Lin JH. Pathologic quiz case: a 39-year-old man with diffuse pleural thickening and massive hemothorax. Epithelioid angiosarcoma of pleura. Arch Pathol Lab Med 2004;128:1299-300.

29. Pramesh CS, Madur BP, Raina S, et al. Angiosarcoma of the pleura. Ann Thorac Cardiovasc Surg 2004;10:187-90.

30. Kimura M, Ito H, Furuta $T$, et al. Pyothorax-associated angiosarcoma of the pleura with metastasis to the brain. Pathol Int 2003;53:547-51.

31. Roh MS, Seo JY, Hong SH. Epithelioid angiosarcoma of the pleura: a case report. J Korean Med Sci 2001;16:792-5.

32. Zhang PJ, Livolsi VA, Brooks JJ. Malignant epithelioid vascular tumors of the pleura: report of a series and literature review. Hum Pathol 2000;31:29-34.

33. Alexiou C, Clelland CA, Robinson D, Morgan WE. Primary angiosarcomas of the chest wall and pleura. Eur J Cardiothorac Surg 1998;14:523-6. 